\title{
Tecnura
}

\section{Efectos de la radiación electromagnética sobre la germinación del maíz}

\section{Electromagnetic radiation effects on germination of corn}

\author{
Alexander Armesto Arenas*, Wilson Angarita**, Ramón Lobo Jácome***
}

Fecha de recepción: 12 de octubre de 2014

Fecha de aceptación: 6 de abril de 2015

Como citar: Armesto Arenas, A., Angarita, W., \& Lobo Jácome, R. (2015). Efectos de la radiación electromagnética sobre la germinación del maíz. Revista Tecnura, 19(45), 65-73. doi: 10.14483/udistrital.jour.tecnura.2015.3.a05

\section{RESUMEN}

En este artículo se evaluó el efecto de radiaciones electromagnéticas sobre la germinación del maíz Zea mays, semilla normalmente cultivada en el municipio de Ocaña, Norte de Santander, Colombia, y cuyo nombre vulgar está descrito como maíz pullita. Se hicieron tres tratamientos, uno con frecuencias de $945 \mathrm{MHz}$ (Megahertz), otros de $440 \mathrm{MHz}$ y la testigo, cada una de estas con tres repeticiones. Dichas emisiones de onda se hicieron con antenas de media onda, situadas a una distancia de 37 centímetros del sustrato donde fueron sembradas las semillas. La radiación electromagnética fue emitida las 24 horas del día, durante ocho días, y los resultados mostraron diferencias estadísticas significativas: a los ocho días se observó un porcentaje de germinación de $92 \%, 80 \%$ y $50 \%$, y a los 15 días de 98 , 95 y $84 \%$, para los tratamientos con emisiones de onda de 945 $\mathrm{MHz}, 440 \mathrm{MHz}$ y las testigos, respectivamente. En este mismo orden se observó un bajo porcentaje de cenizas en comparación con las testigos, con unos resultados de $12,69,16,17$ y $18,45 \%$. Con respecto a la altura, se en los primeros días se encontró una ganancia de longitud para las plantas sometidas a la más alta frecuencia, seguido de las más baja frecuencia y la testigo. Finalmente, en el último día se notó una mayor altura para las de menor frecuencia, seguido de la más alta frecuencia y la testigo.

Palabras clave: electromagnetismo, frecuencia, germinación, Zea mays.

\begin{abstract}
This work evaluated the electromagnetic effects on germination of corn Zea mays, seed usually cultivated in the municipality of Ocaña (North Santander, Colombia), whose common name is described as pullita corn. Three treatments were carried, one with $945 \mathrm{MHz}$ (Megahertz frequencies), other with $440 \mathrm{MHz}$ and a witness one, all of them with three repetitions. Such wave emissions were made with half wave antennas, located at a distance of $37 \mathrm{~cm}$ from the substrate to which the seeds were planted. The electromagnetic radiation emitted was 24 hours a day, for 8 days and the results showed statisticaIly significant differences; observing after those eight days a germination rate of $92 \%, 80 \%$ and $50 \%$, and past 15 days $98 \%, 95 \%$ and $84 \%$, for treatments with wave emissions of $945 \mathrm{MHz}, 440 \mathrm{MHz}$ and the
\end{abstract}

* Ingeniero ambiental, candidato a magíster en Ingeniería Ambiental, Universidad Francisco de Paula Santander Ocaña, Ocaña, Colombia. Contacto: aarmestoa@ufpso.edu.co

** Ingeniero ambiental. Decano de la Facultad de Ciencias Agrarias y del Ambiente, Universidad Francisco de Paula Santander Ocaña, Ocaña, Colombia. Contacto: wangaritac@ufpso.edu.co

*** Ingeniero químico. Docente de la Facultad de Ciencias Agrarias y del Ambiente, Universidad Francisco de Paula Santander Ocaña, Ocaña, Colombia. Contacto: rjloboj@ufpso.edu.co 
witness ones, respectively. Likewise, low percentage of ash was observed in comparison to witnesses, having results of $12,69 \%, 16,17 \%$ and $18,45 \%$. In terms of height, it was observed in the early days, a gain in length for plants subjected to the highest frequencies, followed by the lowest frequency and the witness. For the last day a greater height was noted for lower frequency, followed by the highest frequency and the witness.

Keywords: electromagnetism, frequency, germination, Zea mays.

\section{INTRODUCCIÓN}

La latencia de las semillas tiene su causa en factores externos, ambientales (agua, luz, temperatura, etc.) o en factores internos, propios de ellas (testa dura o impermeable, embrión inmaduro, etc.). Este período puede prolongarse artificialmente, negando a las semillas las condiciones para su germinación. (Ramírez, Romero, \& Henríquez, 1980). Sin embargo, aun en las mejores condiciones de almacenamiento, las semillas sufren una progresiva pérdida de viabilidad, lo cual obliga a realizar controles periódicos para proceder a la regeneración de las muestras si la germinación es menor al 8085\% (Cardoso Almeida, Pita Villamil, \& Gomes de Gouveia, 2000). La energía electromagnética actúa sobre la materia e interrelaciona con los organismos biológicos, en cada etapa de desarrollo desde la germinación, y por ello puede ser una técnica de bajo costo para mejorar la calidad de la semilla. En esta investigación se evaluó el efecto de la irradiación electromagnética sobre la calidad fisiológica de la semilla de maíz Zea mays $L$, del híbrido experimental 'CL-11 x CL-12', con un campo electromagnético de baja frecuencia. Se compararon doce tratamientos de campos magnéticos, con intensidades de 160 y $560 \mathrm{mT}$ (militeslas) y siete tiempos de exposición (t) de 0, 30, 60, 120, 240, 360 y 480 min, en un diseño experimental de bloques completos al azar con cuatro repeticiones. Hubo diferencias estadísticas significativas en la velocidad de emergencia y porcentaje de establecimiento de plántulas al cuarto día, y al día 17 en el peso seco. Se detectó bioestimulación positiva con exposición de 30 y 60 min a una intensidad de 560 y 160 mT (dosis de 705,6 y 27,42 K J m-3 s-1, respectivamente). Con la dosis de 705,6 K J m-3 s-1 hubo un incremento significativo de $69,2 \%$ en comparación con el testigo en velocidad de emergencia, de 90,5\% en establecimiento de plántulas y de $36,6 \%$ en peso seco (Domínguez Pacheco, et al., 2010).

Otros estudios demuestran que la irradiación de semillas con láseres de baja intensidad dan lugar a incremento en la biomasa de las plantas (Stoyanova \& Ivanova, 2000). Un estudio sobre los efectos que la acción de un campo magnético artificial estacionario tiene sobre la germinación de semillas de guisante ha determinado y cuantificado el posible aumento en la velocidad germinativa de semillas de guisante sometidas a la acción de un campo magnético estacionario de $125 \mathrm{mT}$ y $250 \mathrm{mT}$ durante diferentes tiempos de exposición (1 minuto, 10 minutos, 20 minutos, 1 hora, 24 horas y exposición crónica). Los parámetros utilizados han sido: porcentaje de semillas germinadas (Gmáx), tiempo medio de germinación (TMG) y tiempo necesario para que germine el $1,10,25,50,75$ y $90 \%$ de las semillas utilizadas en cada tratamiento (T1, T10, T25, T50, T75 y T90). Del análisis de los datos se puede concluir que la velocidad de germinación de las semillas >sometidas a la acción del campo magnético es mayor que la de las semillas no tratadas (García Rebollar \& Pérez Cabal, 2008). En la India se realizó un experimento para determinar el efecto de la baja dosis de radiación gamma sobre la germinación, el crecimiento, el nitrógeno y la fijación de carbono del cacahuete, en los resultados se encontró que la germinación de semillas se aumentó en un $10-25 \%$ a las dosis más bajas de hasta 5 Gy, mientras que la mejora en vigor de la planta en el 
mismo intervalo de dosis fue mucho más alta (22$84 \%$ ) que las plantas no irradiadas (Singhal, Venu, Ahuja, Singh, \& Kumar Gupta, 2014). La exposición a campos electromagnéticos de $445 \mathrm{MHz}$ y diferentes intensidades de campo en el fríjol mungo Vigna radiata $L$. e Ipomoea aquatica Ipomoea aquatica forsk. En las etapas de crecimiento muestran mejoras en la germinación (Prakob, Jinapang, Wongwattananard, Islam, \& Kirawanich, 2010). Semillas de garbanzo Cicer arietinum L. fueron expuestas en lotes a los campos magnéticos estáticos de fuerza de 0 a $250 \mathrm{mT}$ en pasos de $50 \mathrm{mT}$ durante 1-4 h en pasos de $1 \mathrm{~h}$ para todos los campos. Los resultados mostraron que la aplicación del campo magnético aumenta el rendimiento de semillas en términos de la germinación de laboratorio, velocidad de germinación, longitud de las plántulas y el peso seco de plántulas significativamente en comparación con el control no expuesto (Vashistha \& Nagarajan, 2008). Plantas de tomate Lycopersicon esculentum Mill VFN8 expuestas a campos electromagnéticos de $900 \mathrm{MHz}, 5 \mathrm{~V} \mathrm{~m}-1$ durante un período corto (10 min) muestra que poco después de la exposición, las plantas perciben la radiación como un estímulo nocivo (Roux, et al., 2008). Semillas de girasol sometidas a un campo magnético estacionario de 125 $\mathrm{mT}$ y $250 \mathrm{mT}$ tienen un aumento en la velocidad de germinación, que se pone de manifiesto en una reducción del tiempo requerido para que germine un determinado porcentaje de semillas; este efecto es más pronunciado cuando el tratamiento se realiza de forma crónica (dosis D6 y D12) (Carbonell Padrino, Martínez Ramírez, Flórez García, \& Amaya García de la Escosura, 2005).

Hoy en día se debe reconocer que las empresas agrícolas, en su afán de obtener producciones de una manera más limpia y rápida, están al tanto de cualquier avance tecnológico para favorecerse en tal problemática. Pues bien, uno de esos tantos inconvenientes de siembra y obtención de cosechas es lograr una rápida germinación sin tener ningún efecto en los procesos metabólicos de la planta. Así, aprovechando la energía electromagnética como opción, el objetivo de este trabajo consistió en acelerar la germinación de las plantas de maíz Zea mays por medio de ondas electromagnéticas de entre $945 \mathrm{MHz}$ y $440 \mathrm{MHz}$, con potencia de cinco watts $(5 \mathrm{w})$, emitidas por antenas de media onda, teniendo en cuenta de forma hipotética que las ondas electromagnéticas aceleran la emergencia o germinación de las plantas y que en su efecto dicho campo electromagnético de mayor frecuencia tiene mejor influencia en su respectivo desarrollo. Todo esto con el fin de buscar nuevas alternativas de producción agrícola sustentable y sostenible en pro de un ambiente sano.

\section{METODOLOGÍA}

El ensayo fue hecho en el vivero de la Universidad Francisco de Paula Santander Ocaña en Norte de Santander, Colombia. Se utilizaron 100 semillas de maíz Zea mays por réplica, con un porcentaje de germinación promedio del $85 \%$ y con una masa promedio de 34 gramos, homogeneizadas por tamaño y escogidas al azar, con un total de 900 unidades. En su siembra se utilizó sustrato a base de arenilla y semilleros de plástico, ubicados dentro de jaulas de mallas metálicas a $35 \mathrm{~cm}$ de la antena.

Para la respectiva radiación electromagnética se utilizaron dos circuitos transmisores de $5 \mathrm{w}$ de potencia y dos antenas de media onda con diferente frecuencia (tabla 1 ).

Tabla 1. Descripción de la radiación de las antenas

\begin{tabular}{ccc}
\hline $\begin{array}{c}\mathbf{f} \\
\mathbf{M H z}\end{array}$ & $\begin{array}{c}\mathbf{I} \\
\mathbf{w} / \mathbf{m}^{2}\end{array}$ & $\begin{array}{c}\text { Campo magnético } \\
\mathbf{A} / \mathbf{m}\end{array}$ \\
\hline 945 & $7,5 \times 10^{-3}$ & $4,46 \times 10^{-3}$ \\
\hline 440 & $7,1 \times 10^{-3}$ & $4,33 \times 10^{-3}$ \\
\hline Amperio/metro $(\mathrm{A} / \mathrm{m}) ;$ Intensidad (I); \\
\hline
\end{tabular}

Fuente: elaboración propia.

La intensidad y el campo magnético promedio fueron medidos a 35 centímetros de distancia desde la fuente. 
A partir de la siembra los cultivos empezaron a ser irradiados, durante ocho días las 24 horas del día.

La temperatura promedio del sitio de prueba fue de $21^{\circ} \mathrm{C}$, con una temperatura máxima de 27 ${ }^{\circ} \mathrm{C}$ y una mínima de $17{ }^{\circ} \mathrm{C}$. Dicho experimento fue realizado a cielo abierto y el monitoreo de intensidad de potencia en el respectivo sitio, teniendo en cuenta las diferentes bandas de alta frecuencia, fue de $7,5 \times 10^{-4}$ microwatts $/ \mathrm{cm}^{2}\left(\mu \mathrm{w} / \mathrm{cm}^{2}\right)$.

Todas las mediciones se hicieron con un analizador de espectro de alta frecuencia $(1 \mathrm{MHz}-9,5$ $\mathrm{GHz}$ ) Marca Spectran 60105 - Aaronia.

El diseño experimental que se utilizó fue completamente al azar (DCA) con tres tratamientos: uno con emisiones de onda de $945 \mathrm{MHz}$, otro con $440 \mathrm{MHz}$ y un último sin ningún tipo de radiación electromagnética. Para cada una de estas pruebas se hicieron tres repeticiones.

Para los análisis del comportamiento de las variables (tabla 2) se utilizó el programa Minitab 15. Este es un programa de computadora diseñado para ejecutar funciones estadísticas básicas y avanzadas. Combina el uso de Microsoft Excel con la capacidad de ejecución de análisis estadísticos.
Tabla 2. Variables dependientes e independientes

\begin{tabular}{cc}
\hline Variable dependiente & Variable independiente \\
\hline Germinación & Tiempo $($ días $)$ \\
Altura de las plantas & Frecuencia $(\mathrm{MHz})$ \\
Color & Intensidad $\left(\mathrm{w} / \mathrm{cm}^{2}\right)$ \\
Cenizas & Campo magnético $(\mathrm{T})$ \\
\hline
\end{tabular}

Fuente: elaboración propia.

\section{RESULTADOS}

Con respecto a los resultados se observa que hay mayor porcentaje de germinación en las plantas que presentan mayor frecuencia de radiación (tabla 3).

Tabla 3. Porcentaje de germinación por tratamiento y total de plantas germinadas. Día 8

\begin{tabular}{cccccc}
\hline \multicolumn{6}{c}{ Porcentaje de plantas germinadas } \\
\hline \multirow{2}{*}{ Tratamiento } & \multicolumn{3}{c}{ Repeticiones $(\mathrm{r})$} & Promedio & TOTAL \\
\cline { 2 - 4 } & $\mathrm{r} 1$ & $\mathrm{r} 2$ & $\mathrm{r} 3$ & & \\
\hline 945 & 89 & 98 & 88 & 92 & 275 \\
\hline 440 & 83 & 76 & 80 & 80 & 239 \\
\hline 000 & 53 & 54 & 43 & 50 & 150 \\
\hline \multicolumn{7}{c}{ TOTAL } & & 664 \\
\hline
\end{tabular}

Fuente: elaboración propia.

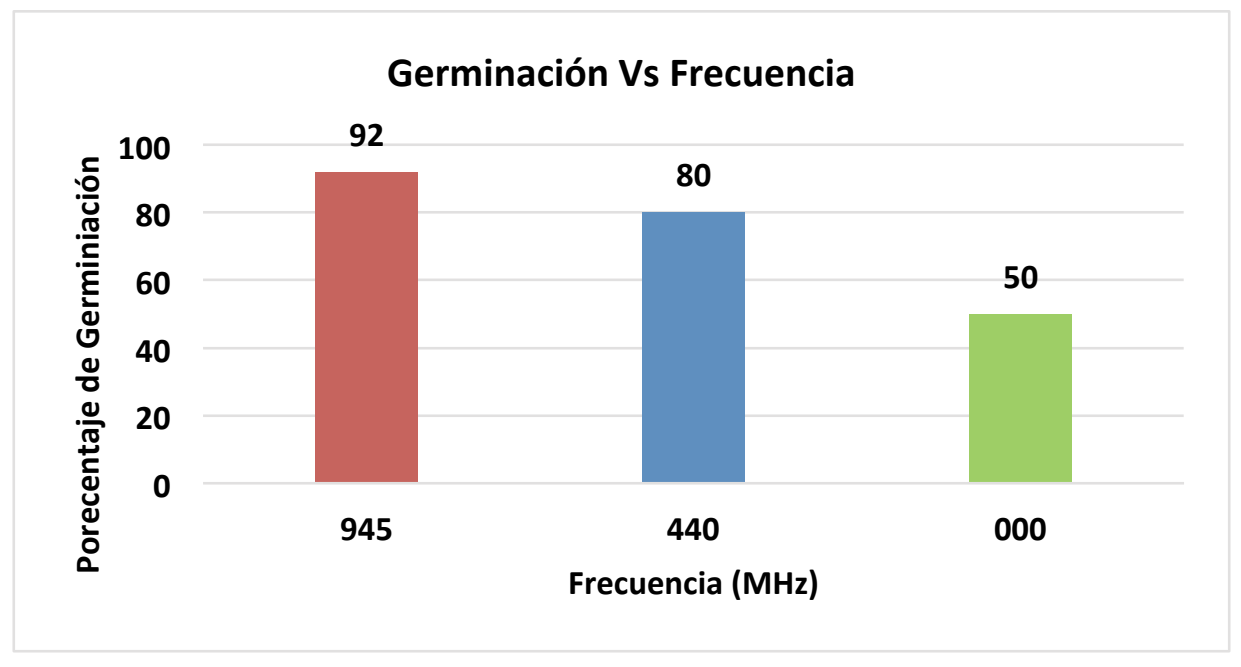

Figura 1. Porcentaje de germinación vs frecuencia. Día 8

Fuente: elaboración propia. 
El tratamiento $945 \mathrm{MHz}$ supera en 12 y $42 \%$ de germinación al tratamiento 440 y $000 \mathrm{MHz}$ (figura 1), y el tratamiento $440 \mathrm{MHz}$ supera en $30 \%$ de germinación al tratamiento $000 \mathrm{MHz}$.

En estos resultados se muestran diferencias significativas entre los tratamientos (tabla 4): las semillas sometidas a radiaciones electromagnéticas de $945 \mathrm{MHz}$ lograron mayor germinación en comparación con las sometidas a $440 \mathrm{MHz}$ y las testigos, de igual forma las semillas sometidas a frecuencia más baja estaban por encima de las pruebas que no fueron sometidas a radiación (testigo).

Tabla 4. Análisis de varianza (Germinación día 8)

\begin{tabular}{|c|c|c|c|c|c|}
\hline Factor & \multicolumn{2}{|c|}{ Tipo } & Niveles & \multicolumn{2}{|c|}{ Valores } \\
\hline Tratamiento & \multicolumn{2}{|c|}{ aleatorio } & 3 & \multicolumn{2}{|c|}{0.440 .945} \\
\hline \multicolumn{6}{|c|}{ Análisis de varianza } \\
\hline Fuente & Gl & Sc & $\mathrm{Cm}$ & $(\mathrm{F})$ & $(\mathrm{P})$ \\
\hline Tratamiento & 2 & 2760,2 & 1380,1 & 51,97 & 0,000 \\
\hline Error & 6 & 159,3 & 26,6 & & \\
\hline Total & 8 & 2919,6 & & & \\
\hline \multicolumn{6}{|c|}{$\begin{array}{l}\text { Varianza }(S)=5,15321, \text { Coeficiente de determinación }\left(R^{2}\right) \\
=94,54 \%, R^{2} \text { (ajustado) }=92,72 \%\end{array}$} \\
\hline \multicolumn{6}{|c|}{$\begin{array}{l}\text { Distribución = F Significancia }=(\mathrm{P}) \text { Cuadrados medios }= \\
\mathrm{Cm} \text {, Suma de cuadrados }=\mathrm{Sc} \text { Grados de libertad }=\mathrm{Gl}\end{array}$} \\
\hline
\end{tabular}

Fuente: elaboración propia.

Teniendo en cuenta que a partir del día ocho las plantas dejaron de ser irradiadas, el día quince (tabla 5) se volvió a hacer un análisis de germinación y se encontró que las plantas sometidas a mayor frecuencia tuvieron mayor porcentaje de germinación.
Tabla 5. Porcentaje de germinación por tratamiento y total de plantas germinadas. Día 15

\begin{tabular}{|c|c|c|c|c|c|}
\hline \multicolumn{6}{|c|}{ Porcentaje de germinación } \\
\hline \multirow{2}{*}{ Tratamiento } & \multicolumn{3}{|c|}{ Repeticiones } & \multirow{2}{*}{ Promedio } & \multirow{2}{*}{ TOTAL } \\
\hline & r1 & r2 & r3 & & \\
\hline 945 & 98 & 98 & 99 & 98 & 295 \\
\hline 440 & 98 & 95 & 93 & 95 & 286 \\
\hline 000 & 87 & 83 & 81 & 84 & 251 \\
\hline \multicolumn{5}{|c|}{ TOTAL } & 832 \\
\hline
\end{tabular}

Fuente: elaboración propia

En este análisis se aprecian diferencias significativas en los respectivos tratamientos (tabla 6), pues el porcentaje alto de germinación se mantiene en las semillas sometidas a la más alta frecuencia, seguido de la prueba a baja frecuencia y la testigo.

Tabla 6. Análisis de Varianza (Germinación día 15)

\begin{tabular}{lccccc}
\hline \multicolumn{1}{c}{ Factor } & \multicolumn{2}{c}{ Tipo } & Niveles & \multicolumn{2}{c}{ Valores } \\
\hline $\begin{array}{l}\text { Tratamiento } \\
\text { Análeatorio }\end{array}$ & 3 & & 0.440 .945 & \\
\hline \multicolumn{2}{l}{ Anas de varianza } \\
\hline Fuente & $\mathrm{Gl}$ & $\mathrm{Sc}$ & $\mathrm{Cm}$ & $\mathrm{F}$ & $\mathrm{P}$ \\
\hline $\begin{array}{l}\text { Tratamien- } \\
\text { to }\end{array}$ & 2 & 360,22 & 180,11 & 33,7 & 0,001 \\
\hline Error & 6 & 32 & 5,33 & & \\
\hline Total & 8 & 392,22 & & & \\
\hline $\mathrm{S}=2,3094, \mathrm{R}^{2}=91,84 \%, \mathrm{R}^{2}$ (ajustado) $=89,12 \%$ \\
\hline
\end{tabular}

Fuente: elaboración propia.

El tratamiento $945 \mathrm{MHz}$ supera en 3\% y $14 \%$ de germinación al tratamiento 440 y $000 \mathrm{MHz}$ (figura 2). Y el tratamiento $440 \mathrm{MHz}$ supera en $11 \%$ de germinación al tratamiento $000 \mathrm{MHz}$. 


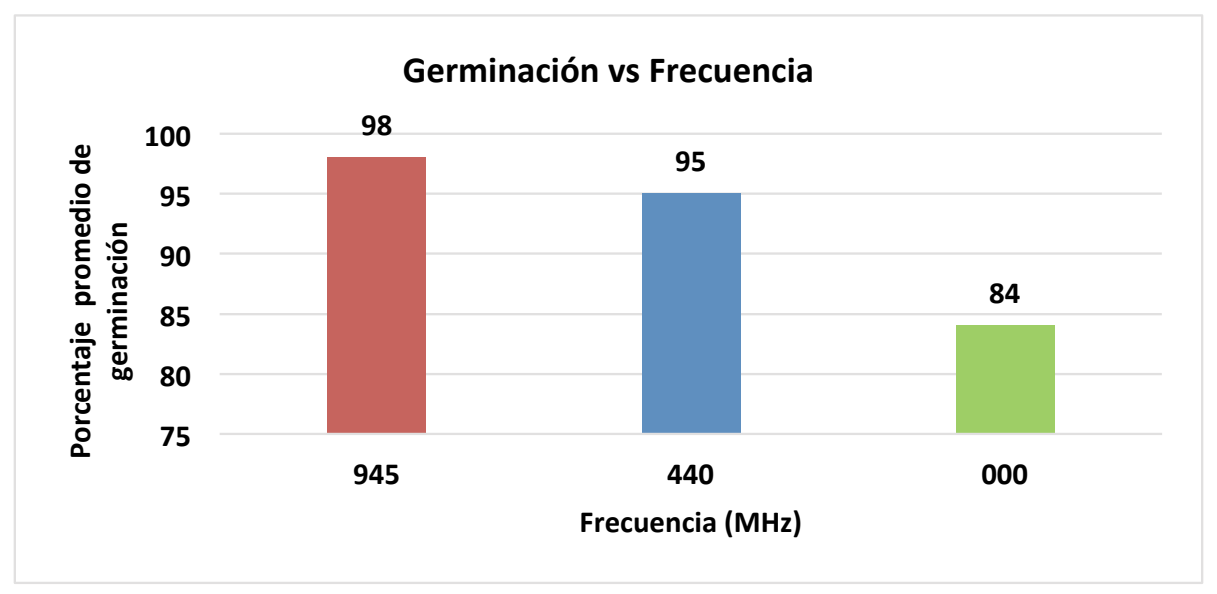

Figura 2. Porcentaje de germinación vs. frecuencia. Día 15

Fuente: elaboración Propia

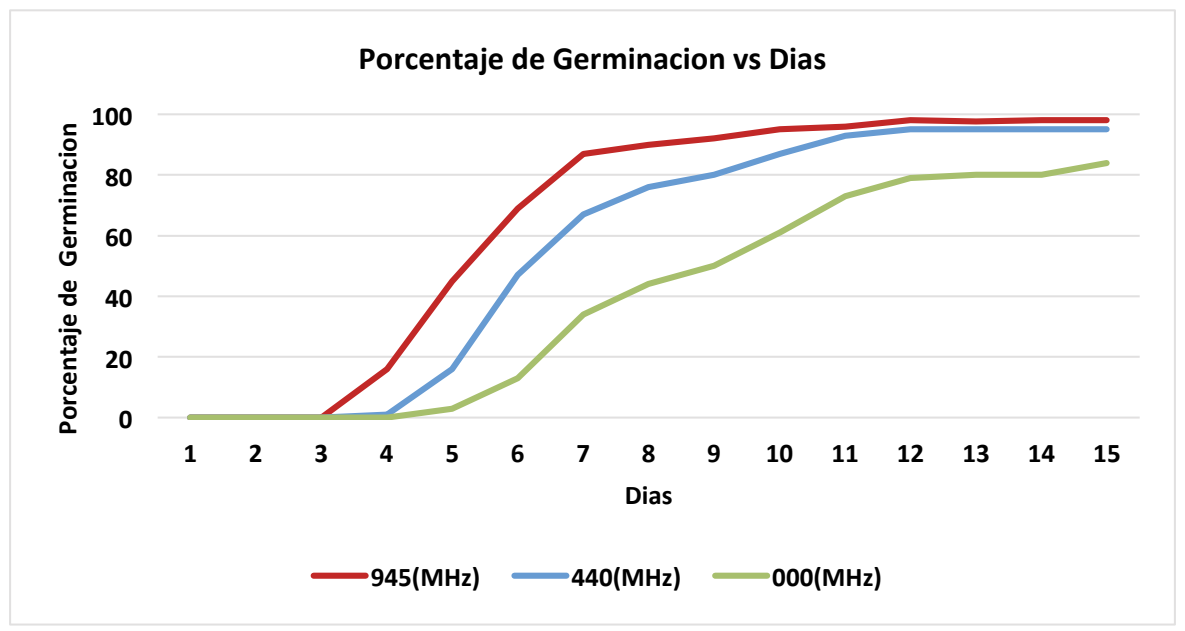

Figura 3. Porcentaje de germinación por día. Día 0 hasta día 15.

Fuente: elaboración propia.

Con respecto a la velocidad de la germinación, se observó que las plantas sometidas a $945 \mathrm{MHz}$ comenzaron primero el proceso de germinación, seguidas de la que recibieron el tratamiento de $440 \mathrm{MHz}$ y $000 \mathrm{MHz}$.

El tratamiento que presentó mayor porcentaje de plantas anormales fue el testigo (tabla 7), y el tratamiento que menos presentó estas características fue el de $440 \mathrm{MHz}$. Para determinar esto se tuvo en cuenta que las plantas anormales formaron parte del número de plantas que germinaron.
Tabla 7. Porcentaje de plantas anormales y que no terminaron su germinación

\begin{tabular}{ccc}
\hline Tratamiento & \% Anormales & \% No Germinaron \\
\hline 945 & 3 & 2 \\
\hline 440 & 1 & 5 \\
\hline 000 & 6 & 16 \\
\hline
\end{tabular}

Fuente: Fuente: elaboración propia. 
De lo anterior se puede observar que las semiIlas sometidas a ondas electromagnéticas de 945 $\mathrm{MHz}$ sobrepasaron el nivel de germinación normal de la planta cuando es cultivada en condiciones normales, teniendo un cuenta que la semillas sometidas a la más alta frecuencia mostraron un color amarillento en el noveno día.

Acerca de la altura en centímetros $(\mathrm{cm})$ promedio que alcanzaron las plantas a los quince días (tabla 8), se observó que las plantas que fueron sometidas a las respectivas radiaciones electromagnéticas estuvieron por encima del tratamiento testigo.

Tabla 8. Altura promedio por tratamiento

\begin{tabular}{lllll}
\hline \multicolumn{5}{c}{ Altura promedio $(\mathbf{c m})$} \\
\hline \multirow{2}{*}{ Tratamiento } & \multicolumn{4}{c}{ Repeticiones } \\
\cline { 2 - 5 } & $\mathbf{r 1}$ & $\mathbf{r}$ & $\mathbf{r 3}$ & Promedio \\
\hline 945 & 11,45 & 13,48 & 11,20 & 12,04 \\
\hline 440 & 12,90 & 12,94 & 13,03 & 12,96 \\
\hline 000 & 9,4 & 10,83 & 10,99 & 10,40 \\
\hline
\end{tabular}

Fuente: elaboración propia.

Las plantas sometidas a $440 \mathrm{MHz}$ superaron en altura (figura 4) a las plantas de $945 \mathrm{MHz}$ y 000
$\mathrm{MHz}$ en $0,92 \mathrm{~cm}$ y $2,56 \mathrm{~cm}$, respectivamente. El tratamiento de $945 \mathrm{MHz}$ superó en altura a las testigos en 1,64 cm.

Con respecto a la altura, se apreciaron diferencias significativas en los respectivos tratamientos (tabla 9): al final de la prueba ganaron mayor altura las plantas sometidas a una radiación de 440 $\mathrm{MHz}$, seguidas de las plantas del tratamiento de $945 \mathrm{MHz}$ y $000 \mathrm{MHz}$. De esto hay que tener en cuenta que por su rápida germinación, las plantas que empezaron ganando altura fueron las sometidas a $945 \mathrm{MHz}$.

Tabla 9. Análisis de varianza (altura alcanzada el día 15)

\begin{tabular}{|c|c|c|c|c|c|}
\hline Factor & & Tipo & Niveles & \multicolumn{2}{|c|}{ Valores } \\
\hline Tratamiento & & aleatorio & 3 & \multicolumn{2}{|c|}{0.440 .945} \\
\hline \multicolumn{6}{|c|}{ Análisis de varianza } \\
\hline Fuente & $\mathrm{Gl}$ & SC & $\mathrm{Cm}$ & $\mathrm{F}$ & $\mathrm{P}$ \\
\hline Tratamiento & 2 & 10,0154 & $4 \quad 5,0077$ & 6,44 & 0,032 \\
\hline Error & 6 & 4,6690 & 0,7782 & & \\
\hline Total & 8 & 14,6844 & & & \\
\hline \multicolumn{6}{|c|}{$\mathrm{S}=0,882138, \mathrm{R}^{2}=68,20 \%, \mathrm{R}^{2}$ (ajustado) $=57,61 \%$} \\
\hline
\end{tabular}

Fuente: elaboración propia.

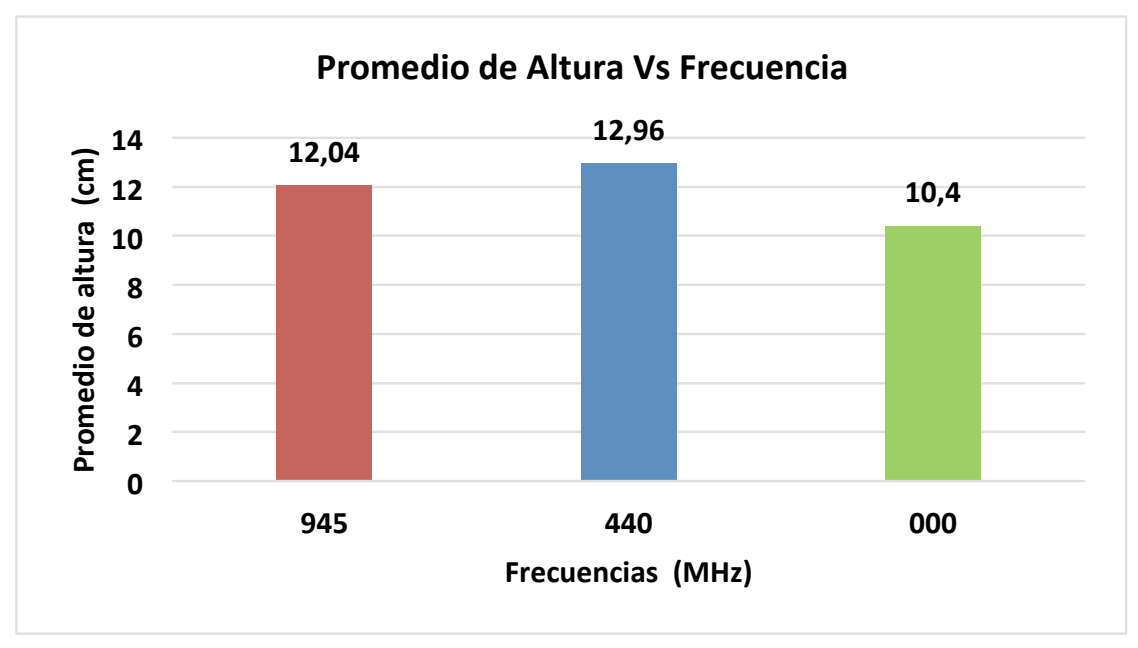

Figura 4. Altura promedio alcanzada el día 15.

Fuente: elaboración propia. 
En cuanto al análisis del porcentaje de ceniza (tabla 10), se encontró que las plantas sometidas a 945 y $440 \mathrm{MHz}$ tuvieron un $5.7597 \%$ y $2.2768 \%$ menos ceniza que las plantas testigo.

Tabla 10. Porcentaje promedio de ceniza

\begin{tabular}{ccccc}
\hline \multicolumn{5}{c}{ Porcentaje de ceniza } \\
\hline \multirow{2}{*}{ Tratamiento Repeticiones } \\
\cline { 2 - 5 } & $\mathrm{r} 1$ & $\mathrm{r} 2$ & $\mathrm{r} 3$ & \% Promedio \\
\hline 945 & 13,7221 & 13,0619 & 11,2869 & 12,6903 \\
\hline 440 & 16,6198 & 13,2126 & 18,6873 & 16,1732 \\
\hline 000 & 19,6015 & 16,0728 & 19,6785 & 18,45 \\
\hline
\end{tabular}

Fuente: elaboración propia.

Estos resultados muestran que las plantas sometidas a $945 \mathrm{MHz}$ y $440 \mathrm{MHz}$ describieron poca diferencia significativa con la testigo (tabla 11), aunque cabe anotar que las plantas sometidas a la mayor frecuencia electromagnética tuvieron menor porcentaje de ceniza.

Tabla 11. Análisis de varianza. Porcentaje de Ceniza

\begin{tabular}{cccccc}
\hline FACTOR & TIPO & \multicolumn{1}{c}{ NIVELES } & \multicolumn{2}{c}{ VALORES } \\
\hline Tratamiento & Aleatorio & 3 & 0.440 .945 \\
\hline \multicolumn{5}{c}{ Análisis de varianza } \\
\hline Fuente & $\mathrm{Gl}$ & $\mathrm{Sc}$ & $\mathrm{Cm}$ & $\mathrm{F}$ & $\mathrm{P}$ \\
\hline Tratamiento & 2 & 50,091 & 25,046 & 5,64 & 0,042 \\
\hline Error & 6 & 26,646 & 4,441 \\
\hline Total & 8 & 76,737 \\
\hline $\mathrm{S}=2,10738, \mathrm{R}^{2}=65,28 \%, \mathrm{R}^{2}$ (ajustado) $=53,70 \%$ \\
\hline
\end{tabular}

Fuente: elaboración propia.

\section{CONCLUSIONES}

Cuanta mayor fue la frecuencia de radiación electromagnética a la que estuvieron sometidas las plantas de maíz, mayor fue la rapidez de su germinación y menor su cenizas. Las plantas de maíz sometidas a radiación electromagnética superaron en número de plantas totales germinadas a las testigos, es decir, la radiación mejoró el porcentaje de germinación de las plantas. Se observó que las plantas sometidas a alta frecuencia de radiación $(945 \mathrm{MHz}$ ) perdieron altura, mostraron un color amarillento en las hojas y debilitamiento en su contextura, lo cual conllevó un estrés a dichas plantas en relación con el aumento de frecuencia.

\section{FINANCIAMIENTO}

El proyecto fue financiado por la Universidad Francisco de Paula Santander Ocaña y orientado desde el Plan de Estudio de Ingeniería Ambiental.

\section{REFERENCIAS}

Carbonell Padrino, M. V., Martínez Ramírez, E., Flórez García, M., \& Amaya García de la Escosura, J. M. (2005). Influencia de campos magnéticos estacionarios de $125 \mathrm{mT}$ y $250 \mathrm{mT}$ en la germinación de semillas de girasol. Ingeniería de Recursos Naturales y del Ambiente(3), 34-39. Obtenido de http:// www.redalyc.org/pdf/2311/231117496006.pdf

Cardoso Almeida, F. d., Pita Villamil, J. M., \& Gomes de Gouveia, J. P. (2000). Efecto de la crioconservación sobre la germinación de semillas de leguminosas. Revista Brasileira de Produtos Agroindustriais, 2, 67-71. Retrieved 2015, from http://www.deag.ufcg. edu.br/rbpa/rev21/Art218.pdf

Domínguez Pacheco, A., Hernández Aguilar, C., Cruz Orea, A., Carballo Carballo, A., Zepada Bautista, R., \& Martínez Ortiz, E. (2010). Semilla de maíz bajo la influencia de irradiación de campos electromagnéticos. Revista Fitotecnia Mexicana, 33(2), 183-188. Retrieved from http://www.researchgate. net/publication/45492504_SEMILLA_DE_MAZ_ BAJO_LA_INFLUENCIA_DE_IRRADIACIN_DE_ CAMPOS_ELECTROMAGNTICOS

García Rebollar, P., \& Pérez Cabal, M. d. (2008). Efecto de la exposición a campos magnéticos estacionarios de $125 \mathrm{mt}$ y $250 \mathrm{mt}$ en la germinación de semillas de guisante. . I Congreso de Estudiantes Universitarios de Ciencia, Tecnología e Ingeniería Agronómica (págs. 93-96). Madrid- España: Escuela Técnica Superior de Ingenieros Agrónomos de la 
Universidad Politécnica de Madrid. Obtenido de http://oa.upm.es/6568/1/Actas_I_Congreso_Estudiantes.pdf

Prakob, P., Jinapang, P., Wongwattananard, P., Islam, N., \& Kirawanich, P. (2010). Growth characteristics of mung beans and water convolvuluses exposed to 425- $\mathrm{MHz}$ electromagnetic fields. Bioelectromagnetics, 31(7), 519. doi:10.1002/bem.20584.

Ramírez, C., Romero, M., \& Henríquez, O. (1980). Estudios de germinacion en semillas de mirtaceas chilenas. Bosque, 3(2), 106-114. Obtenido de http:// mingaonline.uach.cl/pdf/bosque/v3n2/art06.pdf

Roux, D., Vian, A., Girard, S., Paladian, F., Davies, E., \& Ledoigt, G. (2008). High frequency $(900 \mathrm{MHz})$ low amplitude ( $5 \mathrm{~V} \mathrm{~m}$ - 1 ) electromagnetic field: a genuine environmental stimulus that affects transcription, translation, calcium and energy charge in tomato. Planta, 227(4), 91. doi:10.1007/ s00425-007-0664-2
Singhal, R., Venu, B., Ahuja, S., Singh, B., \& Kumar Gupta, V. (2014). ery low dose gamma irradiation stimulates gaseous exchange and carboxylation efficiency, but inhibits vascular sap flow in groundnut (Arachis hypogaea L.). International Journal of Radiation Biology, 90 (2), 179-186. doi:doi:10.3109/09553002.2014.868615

Stoyanova, S., \& Ivanova, R. (2000). Effect of presowing irradiation of seed from winter rapeseed by helium-neon laser on the growth, yield and quality of the green mass. Biotechnology in Animal Husbandry, 16, 75-83. Obtenido de http://cabdirect.org/ abstracts/20003017917.html

Vashistha, A., \& Nagarajan, S. (2008). Exposure of seeds to static magnetic field enhances germination and early growth characteristics in chickpea (Cicer arietinum L.). Bioelectromagnetic, 29(7), 8. doi:10.1002/bem.20426.

\section{(c) $(1) \Theta \Theta$}


